did act as a unit, Group NSp-E would be expected to outperform all other groups, since, for it, both goal and problem materials lead toward solution. That Group NSp-E did not outperform all other groups indicates that these two aspects of the problem situation function independently. Indeed, in the present instance, the tendency to respond to the elevated presentation may have interfered with the tendency to respond on the basis of a goal analysis, and vice versa.

\section{REFERENCES}

BERLYNE, D. E. Structure and direction in thinking. New York: Wiley, 1965.
CAMPBELL, D. T. Blind variation and selective retention in creative thought as in other knowledge processes. Psychological Review, 1960,67, 380-400. COHEN, J. An alternative to Marascuillio's "large sample multiple comparisons" for proportions. Psychological Bulletin. $1967,67,199-201$

DAVIS, G. A. Current status of research and theory in human problem solving. Psvchological Bulletin, 1966, 66, 36-54 deGROOT, A. D. Thought and choice in chess. New York: Basic Books, 1965.

DUNCKER, K. On problem solving. Psychological Monographs, 1945, 58 (Whole No. 270).

K A TON A. G. Eine kle in Auschaungsaufgabe. Psychologische Forschung, 1927, 9, 159-162.

MAIER, N. R. F. Reasoning in humans. III The mechanisms of equivalent stimuli and of reasoning. Journal of Experimental Psychology, 1945, 35, 349-360.

REID, J. W. An experimental study of analysis of the goal in problem solving Journal of General Psychology, 1951, 44. $51-69$.

\title{
Semantic and associative factors in transfer
}

\section{MAY F. D'AMATO and KATHY RUGGER \\ Brooklyn College, Brooklyn, N.Y. 11210}

Two experimental groups learned A-B then A-B'. For both groups, A and B were identical, and $B^{\prime}$ was equally associatively related to $B$ to a low degree. In addition, for one of the experimental groups, $B^{\prime}$ was related in meaning to $B$. Initial transfer results showed significant superiority for the semantically related over the associatively related list. Comparisons with control groups receiving C-D then $A-B^{\prime}$ revealed early positive transfer for the semantically related list and no transfer effect for the associatively related list.

When the $\mathrm{A}-\mathrm{B}, \mathrm{A} \cdot \mathrm{B}^{\prime}$ paradigm is employed, in which $B$ and $B^{\prime}$ are related in meaning, significant positive transfer has been reported (e.g., Slamecka, 1967). It has been suggested that the facilitation in such cases is not due to the semantic relatedness but rather to an underlying associative relatedness (Bastian, 1961). If associative relatedness is assumed to be the effective variable, it would be expected that equal amounts of associative relatedness would produce equal effects with additional semantic relatedness not altering the extent of transfer. The present study seeks to test this hypothesis.

\section{MATERIALS}

Four lists of nine paired associates each were used, two for first-list learning (A-B or C-D) and two for transfer-list learning (A-BS or A-BA). Stimuli were CVCs of low intralist similarity from the $40 \%-73 \%$ level of Glaze (1928). Response items were adjectives or nouns with minimal intralist relatedness. The stimuli in Lists $A-B$ and $C-D$ were equated in Glaze values, while the responses were matched for frequency of usage (Thorndike \& Lorge, 1944), part of speech, and number of syllables. The transfer lists, A-BS and A-BA, were derived by selecting two associates for each response in A-B. These associates were chosen from the norms for discrete free association of Bilodeau \& Howell (1965) such that the associative strength between a response in $A \cdot B$ and each of its two associates was essentially equal. In addition, one of the associates was also judged to have some relatedness in meaning to its corresponding word. For example, food appearing in A-BS and mouse appearing in $\mathrm{A} \cdot \mathrm{BA}$ were associates, each with $p=.06$, to cheese, which appeared in $\mathrm{A}-\mathrm{B}$. Items related in meaning to the first-list responses were used to make up the semantically related transfer list (A-BS), while items merely associatively related made up the associatively related transfer list (A-BA). Thus, the responses of both transfer lists were equally associatively related to their corresponding responses in A-B. The responses in A-BS bore, in addition, a relatedness in meaning to them. For the most part, this semantic relatedness involved synonymity, part-whole relationships, or supraordinate-subordinate relationships. In selecting responses for A-BS and A-BA, Thomdike-Lorge frequency was controlled so that about the same number of AA, A, and 35-49 per million words appeared in each list. The relation between the part of speech involved in A-B and each transfer list was controlled as much as possible. For A-BA, five of the responses were of the same part of speech as the corresponding response in $A-B$, while there were seven such items in A-BS. The actual responses were: List A-B-cheese, color, flower, foot, girl, low, memory, quick, warm; List C-D-belt, country, corner, sleep, salt, kind, magazine, loose, new; List A-BS-food, brown, plant, toe, lady, short, recall, rapid, heat; List A-BA-mouse, bright, pretty, ball, scout, bridge, fond, sharp, soft. The responses in C-D did not appear as associates to A-B responses in the norms of Bilodeau \& Howell (1965). Furthermore, a group of $57 \mathrm{Ss}$, not otherwise participating in the experiment, was presented with a list of 17 words from among which the C-D responses were selected. Four different orders of these 17 words were used in a test of continued association (Bilodeau \& Howell, 1965). Results indicated that, with one exception, the responses in A-B, $A-B S$, and A-BA did not appear among the three continued associates to each response in $C-D$.

\section{SUBJECTS}

A total of 60 volunteers from introductory psychology courses served individually as Ss. There were $15 \mathrm{Ss}$ in each of the four conditions. They were assigned randomly within blocks of four, each condition being represented once in each block.

\section{PROCEDURE}

All lists were presented with a Kodak Carousel slide projector paced by Hunter timers at a $3: 3$ rate with a 6-sec intertrial interval. Four different orders of presentation were used for all lists. Learning was to a criterion of two successive perfect trials for the first list. Transfer lists were presented for five anticipation trials or one perfect trial, whichever occurred last. Instructions presented immediately after first-list learning informed Ss that they were merely to observe the pairs on the very first exposure to the transfer list and to try to anticipate 
them correctly thereafter.

RESULTS AND DISCUSSION First-List Learning

The mean number of trials to criterion for first-list learning showed small variation across the four groups, yielding an insignificant $\mathbf{F}=\mathbf{0 . 5 8}$, $\mathrm{df}=3 / 56$, suggesting comparability of the groups. Since $C-D$ and $A-B$ were matched as closely as possible, it seems likely that they were of equal difficulty.

Transfer-List Learning

The earliest measure of transfer is based on the first transfer trial following the initial observation trial. Mean number of correct responses on this trial produced an $F=5.17$, $\mathrm{d} f=3 / 56, \quad \mathrm{p}<.01$. Specific comparisons were made using the Scheffé test, which required a difference of 2.28 between means for significance at the .05 level. The means were: A-BS, $6.87 ; \mathrm{A}-\mathrm{BA}, 4.40 ; \mathrm{A}_{\mathrm{c}}$-BS (controls receiving $A-B S$ as their transfer list), 4.60; $\mathrm{A}_{\mathrm{c}}-\mathrm{BA}$ (controls getting $\mathrm{A}-\mathrm{BA}), 4.07$, indicating that the difference between A-BS and A-BA was clearly significant, while that between A-BS and its control ( $A_{c}-B S$ ) was just about significant.

Mean correct on the second transfer trial yielded an $F=2.89$, $d f=3 / 56$, $p<.05$. Although A-BS continued to maintain its superiority, Scheffé tests revealed none of the differences to be significant. The means on this trial were: A-BS, $8.07 ; \mathrm{A}-\mathrm{BA}, 6.67 ; \mathrm{A}_{\mathrm{c}}-\mathrm{BS}$, $7.20 ; A_{c}-B A, 6.27$. In Group A-BS, $87 \%$ of the Ss showed no more than one error, while only $47 \%$ did so in A-BA. An index of transfer based on the number of $\mathrm{Ss}$ in each of these groups showing no more than one error vs more than one error revealed a $x^{3}=5.40, d f=1, p<.05$. This test between A-BS and $A_{c}$-BS showed no reliable difference.
On the third transfer trial, A-BS was still superior to all the other lists. However, the mean number correct resulted in an insignificant $\mathrm{F}=0.73$, df $=3 / 56$.

The most sensitive test of transfer effects in this study-mean number of correct responses on the first transfer trial-revealed clear superiority for A-BS over A-BA. Since the associative strength between responses in $A-B$ and the transfer lists was equal for both transfer lists, it must be concluded that beneficial effects based on relatedness in meaning cannot be explained in terms of preexperimental associative relatedness. The experiment doesn't permit a conclusion as to just what produced this result. The exposure time was probably long enough to allow cognitive mediational strategies to be generated, and these may well have developed most rapidly for A-BS. It is possible, however, that the effective mechanism did not involve mediational strategy but, rather, response generalization (Slamecka, 1967). Thus, when a response in $A-B$ was learned to its stimulus, other responses related to it in meaning were automatically strengthened in their connection to that stimulus. The observation trial just prior to the transfer test trials may well have served to make these related responses highly available, as compared to others that might have been even more similar in meaning, and, thus, initially stronger. The plausibility of this explanation is suggested by the fact that on the first (anticipation) transfer trial, 60\% of the Ss receiving A-BS had one or no errors, while the corresponding values for $\mathbf{A}-\mathbf{B A}, \mathbf{A}_{\mathbf{c}}-\mathbf{B S}$, and $A_{c} \cdot B A$ were $13 \%, 13 \%$, and $0 \%$, respectively. It seems unlikely that the one observation trial permitted the development of the necessary cognitive mediational strategies to such an extent, since, it will be recalled, A-BS involved at least three different types of semantic relations relative to $A-B$.

Additional first-trial transfer results are positive transfer for $\mathrm{A}-\mathrm{BS}$ and zero transfer for A-BA. The positive transfer cannot be ascribed to underlying associative relatedness, while the zero transfer for A-BA suggests some beneficial effect was derived from the low degree of associative relatedness present, since, with this paradigm, the use of unrelated responses typically yields negative transfer. Finally, the lack of a difference between $A_{c}-B S$ and $A_{c}-B A$ suggests that the transfer lists were of equal difficulty.

Results for the second transfer trial reveal an absence of positive transfer for A-BS but suggest that a difference between A-BS and A-BA still existed. $B y$ the third trial, any reliable advantage accruing to A-BS had dissipated, undoubtedly due to the fact that all groups showed very rapid acquisition.

\section{REFERENCES}

BASTIAN, J. Associative factors in verbal transfer. Journal of Experimental Psychology, 1961, 62, 70-79.

BILODEAU, E. A. \& HOWELL, D. C. Free association norms by discrete and continued methods. ONR Technical Report, No. 1, 1965. (Contract No. Nonr-475(10), Tulane University.)

GLAZE, J. A. The association value of non-sense syllables. Journal of Genetic Psychology, 1928, 35, 255-267.

SLAMECKA N. J. Transfer with mixed and unmixed lists as a function of semantic relations. Joumal of Experimental Psychology, 1967, 73, 405-410.

THORNDIKE, E. L. \& LORGE, I. The teacher's word book of 30,000 words. New York: Bureau of Publications, Teacher's College, Columbia University. 1944. 